\title{
Il danno da emotrasfusione: dieci sentenze delle Sezioni Unite della Cassazione
}

\author{
Marianna Pulice ${ }^{1}$ \\ 1 Avvocato, Foro di Cosenza

\begin{abstract}
During recent years, health care authorities as well as patients are increasingly concerned about possible transmission virus (HBV, HIV HIV, etc), through a negligent transfusion of infected blood. Until now, despite the extreme importance of this subject, no univocal guidelines have been defined by the Italian Law. Recently the United Sections of the Court of Cassation issued ten judgements concerning cases of malpractice in blood transfusion. This article provides a summary of these legal decisions and describes their implications in clinical practice.
\end{abstract}

Keywords: transfusion of infected blood, negligence, legal decisions

Injury from blood transfusion: ten decisions of the United Sections of the Court of Cassation Pratica Medica \& Aspetti Legali 2008; 2(4): 185-189

\section{INTRODUZIONE}

Il danno da emotrasfusione e da emoderivati è quel pregiudizio subìto da un soggetto a causa di una o più trasfusioni infette $\mathrm{o}$ a causa della somministrazione di emoderivati erroneamente sintetizzati. La dottrina e la giurisprudenza per lungo tempo hanno dibattuto problematiche ad esso correlate senza giungere a posizioni univoche, lasciando così l'operatore del diritto e il personale sanitario privi di punti di riferimento.

Questa situazione di incertezza e lacunosità legislativa può dirsi superata a seguito dell'intervento dirompente delle Sezioni Unite della Corte di Cassazione che hanno emanato ben dieci sentenze (dalla 576 alla 585 del 2008, Tabella I) [1-10], dando delle risposte definitive ad alcune annose questioni sul danno da contagio per via ematica, mentre nel luglio 2008, con la sentenza n. 17975 , si sono occupate dell'ambito operativo della L. 210/1992 [11]. I principi statuiti sono diversi: dal termine iniziale della prescrizione dell'azione di danno nei confronti del Ministero della Salute alla questione della ripartizione dell'onere della prova; dalla natu- ra della responsabilità della struttura sanitaria e del medico ai criteri di individuazione del nesso di causalità.

\section{DESCRIZIONE DEI CASI}

\section{CASO 1}

Tizio si recava presso una struttura sanitaria privata per un intervento chirurgico, durante il quale si rendeva necessaria una trasfusione di sangue. Dopo qualche anno gli veniva diagnosticata un'epatite $C$ e decideva di intentare causa contro il medico e la struttura sanitaria assumendo di aver contratto la patologia a seguito di quell'evento.

\section{CASO 2}

Caio, a causa di una patologia congenita, assumeva degli emoderivati. Dopo qualche anno, a seguito di controlli medici, scopriva di aver contratto 
il virus dell'HIV e presentava la domanda amministrativa volta al riconoscimento dell'indennizzo ex lege n. 210/92, nonché dell'assegno una tantum previsto dalla Legge n. 238 del 1997, art. 1, il quale, però, gli veniva negato.

\section{QUESTIONI GIURIDICHE AFFRONTATE}

\section{CASO 1}

\section{Responsabilità della struttura sanitaria, pubblica o privata, nei confronti del paziente}

La struttura sanitaria all'interno della quale un paziente subisce dei danni, a seguito di una o più trasfusioni di sangue infetto o della somministrazione di emoderivati erroneamente sintetizzati, è responsabile a titolo contrattuale e in via del tutto autonoma rispetto al medico che ha prestato la sua opera. È questo il principio affermato dalle Sezioni Unite della Corte di Cassazione quale organo supremo nell'esercizio della sua funzione nomofilattica, vale a dire quella di mantenere in tutto lo Stato Italiano l'uniformità dell'interpretazione giudiziale.

In effetti, come sottolineato dai giudici superiori, la natura contrattuale della responsabilità è sostenuta da diverso tempo da un orientamento giurisprudenziale che può dirsi consolidato. Laccettazione del paziente, per una visita o un ricovero, comporta di per sé la conclusione di un contratto, definito da alcuni di spedalità e da altri di assistenza sanitaria [12-17]. Quello che è cambiato nel tempo è stato l'iter logico-giuridico con il quale si è arrivati a tale coclusione.

In un primo momento si è individuato il suddetto legame di tipo contrattuale attraverso l'applicazione analogica delle norme del contratto di prestazione d'opera intellettuale normalmente applicate al rapporto medico-paziente e, così, applicate, altresì, al rapporto paziente-struttura sanitaria. Alla luce di ciò, per accertare la responsabilità della struttura occorreva, preliminarmente e inscindibilmente, accertare la responsabilità colposa del medico.

Solo in seguito, nella giurisprudenza più avanzata, si è iniziato a distinguere i due rapporti e considerare quello paziente-struttura ben distinto da quello medico-paziente [18-20].

Tutto questo non ha un rilievo puramente giuridico ma ha ripercussioni immediate nella pratica. Si configurano, infatti, a carico della struttura sanitaria, delle obligazioni proprie e ampie che vanno così a comporre il contenuto di una prestazione alquanto complessa e articolata. Quest'ultima, infatti, vede sì l'obbligazione principale del medico, ma anche una serie di obblighi c.d. di protezione e accessori del personale ausiliario e di tipo organizzativo e infrastrutturale. Non solo: l'approdo giurisprudenziale su tale punto arriva a specificare che tra gli obblighi della struttura vi sono sia quelli specifici, propri della legislazione di settore e sia quelli derivanti dall'art. 1176 c.c., una clausola generale di diligenza dal contenuto ampio e, a volte, sfuggente.

Questa la ricostruzione confermata dalla suprema Corte di Cassazione nella sentenza n. 577/08 con cui i diversi tipi di responsabilità in capo ai diversi soggetti hanno trovato un proprio inquadramento sistematico e logico-giuridico [2].

\section{Ripartizione dell'onere della prova in materia di responsabilità medica}

Nell'ambito di un processo preordinato all'accertamento di una responsabilità medica, passaggio obbligato ma, al tempo stesso, molto delicato, è l'assolvimento dell'onere della prova. La giurisprudenza sul punto è stata piuttosto cospicua.

Tradizionalmente, le dissertazioni sulla responsabilità medica affrontavano il tema della ripartizione dell'onere probatorio facendo ricorso alla distinzione tra obbligazioni di mezzi e obbligazioni di risultato, ormai, però, divenuta inutile.

Scopo di questa distinzione, infatti, era quello di disciplinare e distinguere tra le diverse ipotesi il giusto riparto dell'onere della prova: nel caso di obbligazioni di mezzi l'onere era posto a carico del debitore, nel caso delle obbligazioni di risultato era a carico del creditore.

Con le sentenze n. 589/99 e n. 13533/01 della Cassazione ogni distinzione è stata resa vana in quanto, comunque, in tema di responsabilità medica, è stato previsto che debba essere il medico a dimostrare che si è comportato secondo le leges artis o che il danno non è dipeso da sua colpa. A tal proposito occorre aprire una breve parentesi sul concetto di leges artis. In effetti, nell'accertamento giudiziale proprio di un processo, occorre confrontare la condotta concretamente tenuta dal medico e la condotta che un medico diligente ex art. 1176 c.c. deve tenere. Il contenuto di quest'ultima, però, dipende dalla diversa impostazione del rapporto medico-paziente evolutasi negli anni. Nelle sentenze datate 1925 si incontra la figura di un medico depositario di un arcano sapere, il cui esercizio era insindacabile, salvo errori grossolani, evidenti, madornali. Intorno agli anni '80 la situazione inizia a cambiare, l'atteggiamento sociologico del paziente nei confronti del medico si trasforma fino ad arrivare ad assumere i toni della pretesa. Così, se prima il medico diligente da confrontare col medico reale era un professionista mediocre, oggi deve essere un professionista bravo, 
aggiornato, che conosce le tecniche più avanzate. Dunque, il medico deve dimostrare di aver tenuto una condotta concreta che non si sia discostata dalle regole dettate dalle leggi, dai provvedimenti amministrativi, dalle norme contrattuali e dalle regole tecniche obiettivamente connesse all'esercizio della professione [21].

Per quanto concerne, in particolare, la responsabilità della struttura sanitaria, gli obblighi a suo carico vanno posti in relazione sia agli obblighi normativi esistenti al tempo dell'intervento e relativi alle trasfusioni di sangue, che nel caso de quo erano quelli relativi alla identificabilità del donatore e del centro trasfusionale di provenienza (cd. tracciabilità del sangue), che agli obblighi più generali di cui all'art. 1176 c.c.

A ciò si aggiunga che la sentenza n. 577/2008 ha richiamato, altresì, l'orientamento giurisprudenziale $[22,23]$, secondo cui la difettosa tenuta della cartella clinica non vale ad escludere la sussistenza del nesso eziologico tra la colposa condotta dei medici e la patologia accertata, ma consente anzi il ricorso alle presunzioni, come avviene in ogni caso in cui la prova non può essere data per un comportamento ascrivibile alla stessa parte contro la quale il fatto da provare avrebbe potuto essere invocato (questo è uno dei principi in ordine alla distribuzione dell'onere della prova in cui assume rilievo la "vicinanza alla prova", e cioè la effettiva possibilità per l'una o per l'altra parte di offrirla).

\section{CASO 2}

\section{Ambito di applicazione soggettiva dell'assegno una tantum previsto dall'art. 2 della Legge 210/92}

Una questione finora controversa della Legge $\mathrm{n}$. 210/1992 era l'applicabilità soggettiva dell'assegno una tantum introdotto nella stessa dalla Legge $238 / 97$.

Le Sezioni Unite intervenute con la sent. n. 578/2008 hanno statuito che: «In materia di indennizzi ai soggetti danneggiati da vaccinazioni obbligatorie, trasfusioni ed emoderivati, disciplinati dalla L. n. 210 del 1992, e dalla successiva L. n. 238 del 1997, la previsione di un indennizzo aggiuntivo, sotto forma di assegno "una tantum", ex art. 2, comma 2 della L. n. 210/1992, non è applicabile ai soggetti danneggiati da epatite post-trasfusionale o da infezione HIV, in quanto il predetto dall'art. 2, comma 2, espressamente limita il beneficio a quanti abbiano subito una menomazione permanente alla salute da vaccinazione obbligatoria, senza che sia per questo configurabile una illegittimità costituzionale».

Una tale affermazione di diritto è stata effettuata seguendo un procedimento logico-giuridico che ha tenuto conto sia dell'excursus storico della legge che delle sentenze della Corte Costituzionale n. 226 e 423 del 2000, e dell'ord. n. 522 del 2000. Sin dai primi anni '90 la diffusione di diverse patologie ha assunto dimensioni allarmanti e le conoscenze scientifiche hanno disvelato le loro modalità di diffusione. A tale situazione la risposta dello Stato Sociale Italiano è stata la previsione di una misura di sicurezza sociale di natura assistenziale sotto forma di indennizzo destinato a diverse categorie di soggetti danneggiati. Attualmente l'ambito soggettivo di applicazione è alquanto vasto: sono i soggetti danneggiati da complicanze irreversibili a causa di vaccinazioni, trasfusioni, somministrazione di emoderivati, infezioni per cause da imputare a rischi professionali.

Questa legge ha subìto diverse modifiche nel tempo e, in particolare, sotto la spinta dell'intervento della Corte Costituzionale con la sent. 118/96, è stata introdotta con la L. 238/97 una seconda provvidenza, un assegno una tantum al fine di coprire il periodo tra la manifestazione dell'evento dannoso e l'ottenimento dell'indennizzo (il quale, infatti, decorre dal primo giorno del mese successivo alla presentazione della domanda). La formula legislativa utilizzata, però, per introdurre questa misura è stata ben diversa da quella precedente, infatti non ha richiamato tutti i soggetti previsti per l'applicazione dell'indennizzo.

La Cassazione, con il recente intervento datato gennaio 2008, ha aderito all'interpretazione letterale della norma e, quindi, ha ristretto l'ambito di applicazione soggettivo dell'assegno una tantum rispetto a quello dell'indennizzo dando questa spiegazione: il differente trattamento indennitario è fondato non su una qualità soggettiva delle

\begin{tabular}{|cl|} 
Sentenza n. & \multicolumn{1}{c|}{$\begin{array}{c}\text { Tipologia di infezione contratta in seguito } \\
\text { all'emotrasfusione }\end{array}$} \\
\hline $576 / 2008$ & Infezione da HIV in paziente emofiliaco \\
\hline $577 / 2008$ & $\begin{array}{l}\text { Infezione da HCV in seguito a trasfusioni in } \\
\text { occasione di un intervento chirurgico }\end{array}$ \\
\hline $578 / 2008$ & Infezione da HIV \\
\hline $579 / 2008$ & Infezione da HCV in paziente talassemico \\
\hline $580 / 2008$ & Infezione da HCV \\
\hline $581 / 2008$ & Infezione da HBV, HCV e HIV in diversi pazienti \\
\hline $582 / 2008$ & $\begin{array}{l}\text { Infezione da HCV in paziente sottoposto a } \\
\text { trasfusioni a causa di una gastrite }\end{array}$ \\
\hline $583 / 2008$ & Infezione da HCV in paziente talassemico \\
\hline $584 / 2008$ & Infezione da HIV in seguito a emotrasfusione \\
\hline $585 / 2008$ & Infezione da HCV in paziente talassemico \\
\hline
\end{tabular}

Tabella I. Tipologie di infezioni insorte in seguito alla somministrazione di sangue infetto nelle 10 sentenze esaminate [1-10] 
persone, ma sulla diversa situazione di fatto, da cui discende eziologicamente la patologia indennizzata. In materia di indennizzi a soggetti danneggiati da vaccinazioni obbligatorie, trasfusioni e somministrazione di emoderivati, disciplinati dalla Legge 210/92 e successive modifiche (Legge 238/97), la previsione di un indennizzo aggiuntivo, per il periodo antecedente all'entrata in vigore della L. 210/92, sotto forma di assegno una tantum (previsione ex art. 2, comma 2), non è applicabile ai soggetti danneggiati da epatite post-trasfusionale o da infezioni da HIV, in quanto l'art. 2, comma 2, espressamente limita il beneficio ai soli soggetti dell'art. 1, comma 1 della Legge 210/92.

Ma la Corte di Cassazione, con un intervento ancora più recente, datato 1 luglio 2008, è intervenuta ulteriormente sul tema dell'ambito applicativo della Legge 210/1992, in particolare occupandosi degli emodializzati.

La sentenza 17975/2208 ha stabilito che l'art.1 della legge prevede il risarcimento per i danneggiati da vaccinazioni obbligatorie e per i danneggiati da trasfusioni di sangue e che per trasfusioni di sangue deve intendersi il passaggio di sangue da una ad un'altra persona, o direttamente o previa raccolta e conservazione del sangue e somministrazione dello stesso o di un suo derivato ad un utilizzatore e, dunque, non rientra il prelevamento di sangue da un soggetto e l'iniezione dello stesso sangue nella stessa persona, si tratta in tal caso di una ben diversa autotrasfusione o anche detta circolazione extracorporea.

A fianco di questa argomentazione prettamente tecnica i giudici del palazzaccio si sono soffermati su un aspetto prettamente giuridico che fa leva sulla ratio della Legge n. 210/1992: includere tra i soggetti destinatari del risarcimento ex $\mathrm{L}$. 210/1992 anche gli emodializzati non è una semplice estensione della legge permessa dall'art. 12 delle pre-leggi, ma è un'interpretazione oltre le parole, oltre il contenuto e, dunque, costituisce un'interpretazione analogica non consentita nel nostro ordinamento.

La Legge 210/1992 - viene rimarcato dalla Suprema corte - è un complesso normativo attributivo di benefici tratti da risorse pubbliche e che ne stabilisce, nei primi tre commi dell'art. 1, i rigorosi presupposti. Questi ultimi non consentono di ricomprendere, altresì, coloro che hanno subìto una trasfusione autologa. Nessuna rilevanza viene data al fatto che nella legge non vi è una distinzione in tal senso e che si parla più in generale di "epatiti post-trasfusionali" o comunque che le modalità dei due processi trasfusionali siano sovrapponibili o in ogni modo assimilabili.

Queste considerazioni non hanno impedito però ai giudici di tenere conto del fatto che la letteratura medica sottolinea i rischi di infezioni virali ad essa connessi quando il sangue passa attraverso la macchina perché non correttamente ripulita dalle scorie del paziente che ne ha usufruito precedentemente e così, ai tanti emodializzati che hanno comunque subìto un danno, viene specificato che, "ove si ipotizzi che il danno sia stato provocato dalla macchina sporca, la fonte del risarcimento non è da rintracciare nella L. 210/1992 bensì nella responsabilità contrattuale per danni che lega l'azienda ospedaliera al paziente».

\section{Responsabilità del Ministero della Salute}

Le Sezioni Unite della Suprema Corte, con le sentenze n. 576/2008, n. 579/2008, n. 581/2008 e n. $584 / 2008$, hanno sottolineato il ruolo apicale del Ministero della Salute che non permette di sfuggire alle responsabilità, affermazione che, sul piano operativo, comporta una responsabilità extracontrattuale per danni da emotrasfusioni ed emoderivati.

Nei giudizi sinora instaurati il Ministero ha sollevato costantemente l'eccezione di carenza di legittimazione ma la capacità del Ministero di essere convenuto in giudizio ed essere chiamato a rispondere dei danni da contagio lamentati dall'attore è riconosciuta sia dalla giurisprudenza di merito e di legittimità, che dalla dottrina.

Nelle sentenze, i giudici hanno desunto dalla normativa vigente un preciso obbligo di vigilanza, di direttiva e di controllo, sin dalla sua istituzione con la L. 296/1958, una competenza generale della tutela della salute pubblica e del controllo sui medicinali (ai quali la legge, anche comunitaria, assimila il sangue e i suoi derivati) $e$, in via più specifica, il compito di regolamentare, vigilare, sulle attività trasfusionali relative al sangue umano e ai suoi componenti e per la produzione di plasmaderivati.

Il Ministero ha cercato, altresì, di sostenere la responsabilità del contagio di altri soggetti pubblici, ma tale difesa non ha trovato accoglimento, in quanto l'art. 1 della Legge 296/1958 prevede in capo al Ministero il compito di sovrintendere ai servizi sanitari svolti dalle aministrazioni autonome dello Stato e dagli enti pubblici, prevedendone anche il coordinamento.

Poiché il quadro legislativo di riferimento risulta alquanto composito e corposo, una disamina completa richiede il richiamo di diversi dettati legislativi: L. 592/67; L. 992/67, D.P.R. 1256/71; d.m. Salute del 17.02.72 e del 15.09.72; L. 519/73, L. 833/78; L. 531/87; L. 107/90; L.135/90; d.lgs. 178/91; d.m. Salute del 12.06.91; d.lgs. 502/92; d.lgs. 267/93; d.lgs. 268/93; d.lgs. 449/97; d.lgs. 112/98; L.219/2005.

Di recente, la stessa giurisprudenza di merito si era espressa in tal senso: «Nell'ipotesi di danni cagionati da trasfusione di sangue infetto la legittimazione passiva spetta al Ministero della salute, 
il quale risponde in base alla responsabilità aquiliana di cui all'art. 2043 c.c. e non in base all'art. 2050 c.c. È comportamento colposo anche quello antecedente alla scientifica prevedibilità dell'evento, laddove si dimostri che il rispetto delle normali regole cautelari, comunque, avrebbe impedito il verificarsi di un evento negativo» $[24,25]$.
Le Sezioni Unite hanno ripercorso l'excursus storico-legislativo a partire dall'istituzione del Ministero e dalla conseguente attribuzione delle diverse funzioni, anche in tema di sangue, così chiarendo definitivamente la legittimazione processuale e affermando la responsabilità a carico del Ministero della Salute.

\section{BIBLIOGRAFIA}

1. Corte di Cassazione, SS.UU., sentenza n. 576/08

2. Corte di Cassazione, SS.UU., sentenza n. 577/08

3. Corte di Cassazione, SS.UU., sentenza n. 578/08

4. Corte di Cassazione, SS.UU., sentenza n. 579/08

5. Corte di Cassazione, SS.UU., sentenza n. 580/08

6. Corte di Cassazione, SS.UU., sentenza n. 581/08

7. Corte di Cassazione, SS.UU., sentenza n. 582/08

8. Corte di Cassazione, SS.UU., sentenza n. 583/08

9. Corte di Cassazione, SS.UU., sentenza n. 584/08

10. Corte di Cassazione, SS.UU., sentenza n. 585/08

11. Cassazione civile, sezione lav., sentenza n. 17975 del 1 luglio 2008

12. Cassazione civile, sentenza n. 11316/2003

13. Cassazione civile, sentenza n. 11001/2003

14. Cassazione civile, sentenza n. 3492/2002

15. Cassazione civile, sentenza n. 10297/2004

16. Cassazione civile, sentenza n. 9085/2006

17. Cassazione civile, sentenza n. 1698/2006

18. Cass. Civile, SS.UU., sentenza n. 9556/2002

19. Cassazione civile, sentenza n. 571/2005

20. Cassazione civile, sentenza n. 1698/2006

21. Cassazione civile, sentenza n. $4852 / 99$

22. Corte di Cassazione, sentenza n. 11316 del 21 luglio 2003

23. Corte di Cassazione, sentenza n. 19133 del 23 settembre 2004

24. Tribunale Roma, sez. II civile, sentenza del 3 gennaio 2007

25. Tribunale di Catania, sez. V civile, sentenza n. 266 del 23 gennaio 2007

\section{CORRESPONDING AUTHOR}

Avvocato Marianna Pulice, e-mail: avv.pulice@alice.it 